\title{
Elective Single Embryo Transfer: Future Forward
}

\section{Aamir Javed 1* (D, Dipan Thakkar², Nirmala Agrawal'3 , Snehal Mangaonkar", Rishish Sharma ${ }^{5}$, Suman Saurabh ${ }^{6}$, Dipanshu Sur ${ }^{7}$}

${ }^{1}$ Division of Clinical Embryology, Morpheus Life Sciences, Bengaluru, Karnataka, India

${ }^{2}$ Usha Nursing Home, Nandanvan Society, Anand, Gujarat, India

${ }^{3}$ Juhi Fertility Center, Opposite, Masab Tank, Hyderabad, India

${ }^{4}$ School of Regenerative Medicine, Manipal University, Yelahanka, Bengaluru, India

${ }^{5}$ Indira IVF Hospital, Kanpur, India

${ }^{6}$ AN College, UG Department of Biotechnology, Patna, India

${ }^{7}$ MAGS Medical \& Research Center, Salt Lake, Kolkata, India

Email: ^aamir.javed@morpheusivf.com

How to cite this paper: Javed, A., Thakkar, D., Agrawal, N., Mangaonkar, S., Sharma, R., Saurabh, S. and Sur, D. (2019) Elective Single Embryo Transfer: Future Forward. Advances in Reproductive Sciences, 7, 16-20. https://doi.org/10.4236/arsci.2019.71003

Received: December 11, 2018

Accepted: January 14, 2019

Published: January 17, 2019

Copyright $\odot 2019$ by author(s) and Scientific Research Publishing Inc. This work is licensed under the Creative Commons Attribution International License (CC BY 4.0).

http://creativecommons.org/licenses/by/4.0/

\begin{abstract}
Notwithstanding the most noteworthy verifiable live birth achievement rates for couples experiencing in vitro fertilization (IVF), there has been a scourge of iatrogenic twin and higher gestation development considered from this treatment. While some expanded peril is natural for the barren populace requiring treatment, the act of multiple embryo transfer adds to these inconveniences and results, particularly concerning its part in higher number pregnancies. Improvement in cryopreservation procedures has permitted conservation of supernumerary embryos for use in future cycles, and refinements in culture frameworks and embryo selection have brought about the transfer of elective single embryos while keeping up positive pregnancy rates. The willful exchange of a single top notch quality elective single embryo transfer e-SET (Blastocyst) has fundamentally lessened the multiple gestation rates and boosted the rate of singleton pregnancy without compromising the global success rates. We acknowledge that in high-risk situations (e.g. previous history of preterm gestation and poor maternal wellbeing), double-embryo transfer (DET) or triple embryo transfer (TET) ought to be disallowed because of unsuitably high perils. Be that as it may, we contend that ordering e-SET for every single young lady can be viewed as an unsatisfactory break of patient self-governance, particularly since DET/TET offers certain ladies money related and social focal points.
\end{abstract}

\section{Keywords}

IVF/ICSI, Multiple Gestation, e-SET, DET, TET 


\section{Introduction}

Elective single-embryo transfer (e-SET) is a procedure in which one top quality embryo is selected from a larger number of existing embryos (cleavage stage or blastocyst), and is placed in the uterus. The embryo selected for e-SET might be from a prior IVF cycle (e.g., cryopreserved embryos (frozen)) or from the present fresh IVF cycle that yielded more than one embryo. In vitro fertilization (IVF) treatment globally is confounded by a high rate of multiplex gestational pregnancies. The Society for Assisted Reproductive Technology announced that $23 \%$ of ladies $<38$ years old with a pregnancy from their IVF treatment had a twin-gravid pregnancy in 2014 [1]. Despite the fact that this is a change over past years, it remains an inadmissibly high rate of twins, considering the restorative dangers and fiscal hardship related with such pregnancies. Mersereau et al. (2017) have added robust cornerstone help to the conclusion that elective-single embryo transfer (e-SET) is very viable at diminishing multiplex-gestation birth rates: a $47 \%$ lessening with the utilization of e-SET contrasted and double-embryo transfer (DET) and triple-embryo transfer (TET). Besides, utilizing information from their investigation and others, Mersereau's group has driven an update of American Society for Reproductive Medicine advisory group conclusion rules to unambiguously call for e-SET for ladies younger than 38 years with an ideal forecast for pregnancy [2].

\section{Current Verdicts}

With the expanding knowledge of confirmation and expert dexterous rules, why is DET or TET still so normal globally? In their article, Mersereau et al., report a complete examination of 10 years of national e-SET information, finding a 10\% $15 \%$ diminishment in live birth rate (LBR) and a $47 \%$ decrement in multiple birth rate with the utilization of e-SET (Blastocyst) [2]. This reduction isn't appealing to either doctors or patients, for whom IVF pregnancy rates matter an extraordinary arrangement. In reality, we have demonstrated that, regardless of instruction about the dangers of twins after DET or TET, most patients would in any case pick this alternative over e-SET, even with as meager as a 10\% drop in the LBR [3]. However we believe that the $10 \%-15 \%$ contrast in LBR might be an overestimate of the negative impact of e-SET on LBR, considering patterns in current clinical IVF practice. As revealed, blastocyst transfers are widening up progressively, and e-SET live birth rates are higher with blastocyst than with cleavage-stage embryos [2]. Actually, the LBR contrasts amongst TET, DET and SET were as yet decreased, however just in the $6 \%-8 \%$ marginally, when taking same cycle transfer of blastocyst in the first or second cycle. Indeed, even this might be an overestimate of the genuine distinction amongst TET, DET and SET, on the grounds that higher pregnancy rates are seen when the single transferred embryos originates from a bigger accomplice of accessible available embryos [4]. 


\section{Controversy: Is e-SET Appropriate for Me?}

Research surveys the odds of progress (pregnancy and live birth) in view of the denominator of embryos transferred during an ART practice. These research institutes that among women with a good possibility of conception with ART, those who chose to have a single embryo placement had alike figure of live-birth rates correlated to those who elect to transfer multiple embryos, but almost all of the newborn they delivered were singletons. However, the guideline for the number of embryo/s transferred were conceptualized by the Practice Committees of the American Society for Reproductive Medicine (ASRM) and the Society for Assisted Reproductive Technology (SART) [5] [6], stating single elective embryo transfer should be practiced for the patients with a positive prediction generally women aged 35 years or below with a good quality eggs and embryo/s. These rules have been created to help doctors with clinical embryologist in regards to the care of their patients. They are not planned to be a convention to be connected in all circumstances, and can't substitute for the individual judgment of the treating doctors in view of their insight into their patients and particular conditions.

\section{Discussion}

In this manner, it is likely a false correlation which judge the accomplishment of SET with at least one or more embryos cryopreserved (no less than two embryo cryopreserved in the adherent) against DET with at least one or more embryo cryopreserved (at least 3 embryos in the adherent). In an ongoing investigation of national IVF results information, we firmly managed for the size of the accessible group and found very similar pregnancy rates in more youthful and young patients experiencing elective e-SET versus DET on day 5 - 6 [4]. We imagine that this pattern of expanding blastocyst transfer joined with enhancements in developing topnotch embryo selection systems, (for example, pre-implantation genetic screening) will bring about further increments in e-SET pregnancy rates and enable centers to all the more unhesitatingly offer e-SET with next to zero effect on their facility particular pregnancy results [4]. Regardless of proceeding with specialized advances, in any case, it is likely that little however potentially noteworthy LBR contrasts will hold on amongst e-SET and DET if as a field we proceed to report and underscore pregnancy rates per exchange rather than total pregnancy rates per fresh IVF cycle. As said in Mersereau et al.'s. Paper, prescient models recommend that total LBRs with the utilization of consecutive e-SET are equivalent or better than DET. Additionally considers affirming this forecast will persuade doctors, patients, and assurance providers of the advantages and plausibility of e-SET, regardless of whether this system requires extra exchanges and a marginally longer time to pregnancy. A sound singleton conveyance ought to be the objective of all IVF cycles, and this is best accomplished by e-SET [7].

\section{Future Aspects}

Difficulties in expanded utilization of eSET exist. These incorporate service pro- 
vider, patient education, financial contemplations, embryo selection and effective cryopreservation.

\section{Conclusion}

The past couple of decades have seen the development of IVF as the best level treatment for infertility. Technological improvements, for example, pre-implantation genetic screening (PGS), improvement in cryopreservation methods, extended embryo culture with blastocyst selection may at last lessen the requirement for DET/TET by giving ideal pregnancy rates from the elective transfer of a single embryo. Advances in reproductive medicine should go for the best quality level IVF result to be a singleton term live birth pregnancy with e-SET. The future achievement of ART lies in elective single embryo transfer, future forward.

\section{Acknowledgements}

I acknowledge the colleagues of division of clinical embryology, morpheus life sciences Bangaluru, India for their collaboration.

\section{Conflicts of Interest}

The author/s declare that he has no competing interests.

\section{Ethics Approval and Consent to Participate}

Not applicable.

\section{Consent for Publication}

Not applicable.

\section{Availability of Data and Material}

The datasets supporting the conclusions of this article are included within the article.

\section{Funding}

The project was a non funded project.

\section{Authors' Contributions}

AJ imagined the idea of the assessment with their co authors and took an interest in its plan, coordination and drafted the original copy.

\section{References}

[1] Practice Committee of the American Society for Reproductive Medicine-Practice Committee of the Society for Assisted Reproductive Technology (2017) Guidance on the Limits to the Number of Embryos to Transfer: A Committee Opinion. Fertility and Sterility, 107, 901-903. https://doi.org/10.1016/j.fertnstert.2017.02.107

[2] Mersereau, J., Stanhiser, J., Coddington, C., Jones, T., Luke, B. and Brown, M. 
(2017) Patient and Cycle Characteristics Predicting High Pregnancy Rates with Single Embryo Transfer: An Analysis of the SART Outcomes between 2004-2013. Fertility and Sterility, 108, 750-756. https://doi.org/10.1016/j.fertnstert.2017.07.1167

[3] Ryan, G.L., Sparks, A.E., Sipe, C.S., Syrop, C.H., Dokras, A. and Van Voorhis, B.J. (2007) A Mandatory Single Blastocyst Transfer Policy with Educational Campaign in a United States IVF Program Reduces Multiple Gestation Rates without Sacrificing Pregnancy Rates. Fertility and Sterility, 88, 354-560. https://doi.org/10.1016/j.fertnstert.2007.03.001

[4] Mancuso, A.C., Boulet, S.L., Duran, E., Munch, E., Kissin, D.M. and Van Voorhis, B.J. (2016) Elective Single Embryo Transfer in Women Less Than Age 38 Years Reduces Multiple Birth Rates, But Not Live Birth Rates, in United States Fertility Clinics. Fertility and Sterility, 106, 1107-1114.

https://doi.org/10.1016/j.fertnstert.2016.06.017

[5] Society for Assisted Reproductive Technology.

https://www.sart.org/patients/a-patients guide to-assisted-reproductive-technology/

[6] American Society for Reproductive Medicine.

https://www.asrm.org/news-andpublications/practice-committee-documents

[7] Javed, A., Yesudhas, S., Agarwal, D. and Shrotriya, R. (2018) Prologue: Juvenility Inferences of Parental Advance Aging. Advances in Reproductive Sciences, 6, 109-112. https://doi.org/10.4236/arsci.2018.63009

\section{Abbreviations}

e-SET: elective single embryo transfer,

DET: Double embryo transfer,

TET: Triple embryo transfer,

LBRs: Live birth rates,

IVF: In vitro fertilization. 